\title{
Susanne Benzler Justiz und Anstaltsmord nach 1945
}

Die erste ausführliche Dokumentacion der „Euthanasieaktion* des Dritten Reiches (und zugleich des Nürnberger Ärzteprozesses) erschien 1949 unter dem Tirel "Wissenschaft olıne Menschlichkeit «.' Die Geschichte dieses Buches ist symptomatisch für die Auseinandersetzung mit den Morden an psychisch Kranken nach 1945.'

Der $\{1$. Deutsche Ärztecag beschloß im Okrober 1948 dic (auch finanzielle) Untersrützung einer Dokumentation des Nürnberger Ärzreprozesses. Trotz dieses Beschlusses, der die ernsthafte Intention der Ärzteschaft zur Beschäftigung mit ihrem Beitrag zum nationalsozialistischen Unrechusstaat verdeuclichte, fand sich kein Arzt bereit, die Dokumentation zu erstellen. Ein Privardozent (A. Mitscherlich) und ein Student (!) (F. Mielke) übernahmen schließlich diese Aufgabe. Die 10000 Excmplare der Schrift, die 1949 an die Ärzte verteilt wurden, riefen keinerlei öffentliche Reakrion - keine Rezension, keine Leserzuschriften - hervor. In den folgenden Jahren schien niemand das Buch zu kcnnen. Erst 1960 wurde es wieder aufgelegt. Zwar erschienen in den 6oer und 7oer Jahren einige Untersuchungen zur »Euthanasieaktion ${ }^{3}$, im großen und ganzen fand die Auseinandersetzung mit diesem NSMassenverbrechen jedoch fast ausschließlich im Gerichtssaal statc. An der Argumentation der Gerichcsurteile zum Tatkomplex "Euthanasie läßt sich unschwer erkennen, daß die juristische Auseinandersetzung mit ihm eine ähnliche Stoßrichtung hatte wie die öffentliche Tabuisierung des Themas: Überwiegend wurden die Täter exkulpiert. Diesc Exkulparion durch die Rechtspraxis ordnete sich fast bruchlos ein in die Restaurationsbestrebungen konservativer Rechtslehre, die ndie Beteiligung bürgerlicher Funktionsgruppen (Richter[n] und Ärzte[n]) an der Terrorpraxis des NS-Staztes in weitem Maße der Ahndung [zu] encziehen $*^{4}$ sucheen. In den letzten Jahren haben sich vor allem Ernst Klce und die Autoren der Zeirschrift "Beiträge zur nationalsozialistischen Gesundheits- und Sozialpolitik « um die Aufhellung der Geschichte der Anstaltsmorde bemüht.' Eine zusammenhän-

I Die Broschiure "Das Dikıa der Menscbenverachtung erschicn 1947 noch wahirend des Äracprozesses und ist ein Vorläufer des genanncen Buches.

2 Vgl. die Darstellung bes Mitscherlich, A./Mielke, F. (Hg.): Medizın ohne Menschlichkerr - Dokumente des Nürnberger Ärzteprozesses, Frankfur 1978, S. 14 f.

3 Honolks, B.: Dic Kreuxclschrcibar - Äralc ohne Gewissen - Euchanasıe im Drucen Reich, Hamburg 1961; Schmidi, G.: Selektion in der Heilansiale 1935-1945 (1965). Frankfun 1983; Raul, F. K.: Naztmordakuon $\mathrm{T}_{4}$ - Ein Berıche über dic erste industriemäßig durchgeführe Mordakeion des Niziregimes, Berlin (O) 1973 - in der Bundesrepublik erschienen uncer dem etwas arreführenden Oberiel: Die Psychiarie in Serudel der Euthanasie, Frankfurt 1979; Schmulil, H. W.: Rassenliygene, Nationalsozialismus, Euthanasic. Von der Verhütung zur Vernichtung *lebensunwereen Lebens* 1890-1945. Götningen 1987.

4 Perels. 3.: Die Reseaurauon der Recheslchre nach 1945, un: KJ 4/19\$4, 5. 359 if.; Zieat S. 360 f.

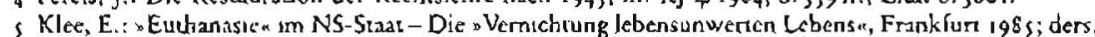
$\left(\mathrm{Hg}_{\mathrm{g}}\right)$ : Dokumente zur "Eurhanasse", Frankfure 1985; Aly, G. u. a.: Aussonderung und Tad - Dic 
gende Untersuchung zur juristischen Aufarbeitung der «Euthanasieaktion* seit 1945 feblt jedoch bis heute. ${ }^{6}$

Im folgenden soll die juristische Angumentation der "Eurhanasie -Urteile zwischen 1945 und 1974 dargestellt werden. Die innere Entwicklung der Rechtsprechung vollzog 1947/48 eine Wendung um i 80 Grad; von der Ahndung des Anstaltsmord's zur weitgehenden Exkulpation der „Euchanasie«-Täter. Dargestelle werden daher im ersten Teil die Rechrsprechung der Jahre 1946/47, die beispielhaft zeigr, mit welchen Argumentarionen eine Ahndung der "Euthanasie -Tacen möglich war. Im zweiten Teil werden diejenigen juristischen Theorien analysiert, die zur Exkulpacion der Täter dienten.

\section{Erste Pbase: 1946-1947/48 Verurteilung der "Euthanasie"-Tater}

Bis 1933 hatte der Staat im wesentlichen als Garant des Rechts gegolten. Mit dem Machtantritt der Nazis verkörperte er das Unrecht selbst und leitere zur Teilnahme an seinen verbrecherischen Taten an oder vereinfachre ihre Durchführung. Diese veränderte Stellung des Staases im Nationalsozialismus ließ nach 1945 Tärerschaft in einem neuen Licht erscheinen: der Tater war nun "gekennzcichnet gerade nicht durch den Widersprucl zu der Idee des damaligen Staaces, sondern durch den Einklang mit ihr . ... ${ }^{7}$

Wie war die strafrechtliche Verantwortlichkeit von Personen zu beurteilen, die nicht als Außenseiter der Gesellschaft und entgegen staaclichen Normen, sondcrn mir staatlicher Anleitung und Billigung hunderte, oft tausende Menschen getöter hatten? Dic Beantwortung dieser Frage, die das grundsätzliche Verhälınis der Justiz zum Unrechtsstaat des Nationalsozialismus berühr, unterschied sich in der ersten Phase - 1946-1947/8 - grundsätzlich von der der späteren, erwa mit der Gründung der Bundesrepublik begrnnenden Phase.

\section{Rechtswidrigkeit der „Euthanasieaktion"}

Die Massentötungen im Rahmen der „Euthanasieaktion « wurden durch eine Großorganisation in Gang gesetzt, die ihre scheinrecheliche Legitimation durch eine Ermächtigung Hitlers vom Okrober 1939/r.9. 1939 zur Törung »unheilbar Kranker« erfuhr. Da die Befolgung von Geserzen unter rechtsstaaclichen Verhältnissen die Strafbarkeir in der Regel ausschließe, mußten die Richter der ersten "Euthanasie«-Verfahren zunächst prüfen, ob die "Gnadentodu-Akcion rechtstvidrig war, obwohl sie durch ein Schreiben des damaligen Inhabers der Staatsgewalt, Adoif Hitler, abgesichert schien.

klinssche Hinrichrung der Unbrauchbaren (Bcıü̈ge xur natsonalsozıalistischen Gesundlieics- und Soztalpoliuk 1), Berlin 398 ; dies.: Relorm und Gewissen - Euthanassen Im Dienst des Fonschnas (Beitrage zur nationalsozialisuschen Gesundhetes- und Soxialpolitik 2), Berlin 1985.

6 Wichuge Vorarbetcen hicrzu sind: Brokmeicr, P.: Die Vorseufe der Endlösung - Zum Frankluner Euthanasseprozeß 1967/68, in: Gewerkschaftliche Monatshefte 21 (1970), S. 28 ff; Gnuchmann, L.: Euchanasse und Justrz im Dnten Reich, in: Vierreljahreshelte lür Zeirgeschichte 20 (1972), S. 23 sff.; Kramer, H.: Oberlandesgenchıspräsıdenten und Generalstaatsanwalte als Gehilfen der NS- Euthanasıe", In: KJ r/1984, S. 2s fff.; Klee, E.: Was sie taten - was sie wurden. Ärzte, Jurısten und anderc Beceiligıe am Kranken- und Judenmord, Frankfurt 1986; Fnedrich, J.: Dic kalte Amnesuc - NS-Täıcr in der Bundesrepublik, Frankfurt $1984,5.179 \mathrm{ff}$.

7 Lange, R.: Anmerkung zu der Encscheidung des KG Berlin von 24. 8. 1946, un: DRZ 1947. S. i 98 if.; Zical S. 200 r. Sp 
Die Rechisprechung der ersten Jahre, die wesentlich durch die Urteile der Frankfurter und Berliner Gerichte geprägt wurde, ${ }^{3}$ stellte die Rechtswidrigkeit der Ermächtigung vom 1.9. 1939 eindeurig fest. Damit erfültten die Taten, die im Rahmen der "Euthanasieakrion « begangen worden waren, in der Regel den objektiven Tatbestand des $\$ 21 \times$ SrGB?

Begründet wurde die Rechrswidrigkeit mit "rechrspositivistischen $b z$. formellen und naturrechtlichen bzw. materiellen Argumenten.

Dem Hitierschen Ermächrigungsschreiben wurde vom OLG Frankfurc bereits aus formellen Gründen jede Rechrsgültigkeit abgesprochen:

„Das Ermächtigungsschreiben Hitlers vom 1.9.1939 was keine gültige Rechtsnorrm. Es rrägt weder am Kopf des Schreibens noch bei der Unterschrifr cinen die statsrechrliche Srellung des Unterzeichneten bezeichnenden Zusatz, noch die Gegenzeichnung eines Ministers, noch ist es veröffentlicht worden. Die im nationalsozialistischen Schriftum vertretene These, daß jeder Willensïußerung Hitlers Gesetzeskraft zukomme, ist zu keiner Zeit selbst Gesetz geworden. Bis zuletzr blieben auch während der nacionalsozialisusehen Herrschaft die Gegenzeichnungen durch den zuständigen Minister und dic Verkündung im Reichsgeserzblat Brauch. a ${ }^{\text {to }}$

Das LG Freiburg verwies auf das SrGB, das auch während des Nationalsozialismus Euthanasie im eigentlichen Sinne und Massenmord nicht zuließ:

* Auch unter der Herrschaft des Nationalsozıalismus war dic Törung von Gcistcskranken als Mord zu beureilen; vgl. ... Komm. zum StGB 12. Aufl. 1944, Anm. 4 zu \211 S.977.4"

Das LG Frankfurt entwickelte demgegenüber - weil es die formelle Rechrswidrigkeit des Hitler-Schreibens wegen der möglicherweise bestehenden "normativen Kraft des Faktischen " nicht zweifelsfrei feststellen wollte ${ }^{12}$ - eine naturrechtliche Argumentazion, die der Ermächrigung aus maceriellea, inhajelichen Gründen die Legitimation enczog:

.Wenn ... aus Gründen der Rechissicherheit auch grundsäzzlich zugestanden werden muß, daß Im allgemewnen das Gesetz ohne Rücksiche auf seinen Inhaje als rechesverbindlich angesehen werden muß, so finder dieser Satz doch unter allen Umsiönden seine äußerste Begrenzung darin, daß die Staztsfübrung nichı willkürlich bestirnmen kann, was Recht oder Unrecht ist. Der Start ist nienals die alleinige Quelle des Rechtes. Auch er selbst ist dem Recht unterworfen, auch er ist an die ewigen Normen des narürlichen Sittengesetzes gebunden, und verletze cr sie, so enrbchre sein Geserz der verpflichrenden Krafe für alle Unterancn.

Dieses sogenannte Gesetz Adolf Hitlers verletzte aber das elementarste aller Naturrechtsgütcr, das Recht des Individuums auf Leben, das der Staat als Kultumation nur auf Grund eines Richeerspruchs oder im Kriege fordern darf. Es verlerzte alle Grundsïlze von Situichkeit und Menschlichkeit und verleugnetc die Errungenschafeen ciner zweitausendjährigen Kulzur. Es verstieß gegen die Grundlchren des Christentums und sprach allem Hohn, was bisher als Recht und Sitte galt. Das Recht auf Leben aber ist cin unentbehrlicher Teil jeder sittlichen Gemeinschaftsordnung. Die siulich und auch rechtlich verbindliche Kraft der Naturrechtsnorm: :Du solist nicht töten ‘ isı deshalb so scark, daß sie nicmals von der formellen Rechiskraft eines Gesetzes verdrängt werden kann. Dieser Erlaß Hitlers hat somit kein Rechr geschaffen, sondem nur das Unrecht in seiner krassesten Form, höchstenfalls zum formell gültigen Rechessatz crhoben, auf den sich aber wegen seines Unrechesgehalts niemand berufen kann. Ein Gesezz mit so elementar unsiulichem Inhalı ist immer und unter allen Umständen wegen dicses Inhales rechesungültig. Daraus ergibe sich, daß das angebliche Geserz rechısunwirksam

8 LG Berlin 25-3.1946, abgedruckt in: Rücer, C.F. (Hg.): Justiz und NS-Verbrechen - Sammlung deutscher Serafureile wegen nauonalsozialisuscher Tötungsverbrechen 1945-1966. Amsicrdam $1968 \mathrm{ff} ., \mathrm{Bd} .1, \mathrm{~S}$. 33 fl. Die folgenden Urtesle z'erden, sowett sic un der 0. 1. Urteilssammlung aufgefuhr sind, mit Bandnr. und Sestenzahl angezeber. (2. B: LG Berlin 15.3.1946, I $33(1$.$) : KG Berlin$ 24.8. 1946, I 39 lf., LG Frankfure 21. 12.1946.1 I 39ff., 30.1.1947, I 231 fl., 9. 2. 1949, IV 47 ff,; OLG Frankfun 12.8.1947. I 166 If, 36. 4. 1948, I 262 ff., 20. 10. 1948, 136611,2$\} .6 .1949$. IV if if.

9 Ich gebe nur auf die Aburcilung nach dem SiGB cn, die Abureilung nach dem Kontrollrasageselz Nr. 10 fand nur in wenigen Ausnahmes statt.

10 OLG Frankfun 12.8.1947, 1:71.

is LG Freiburg $16.11,1948$, V1 484 fl., ZiLat V1 sto

i2 LG FrankJure 21. 12.1946, I is 6 und LG Frankfure 10. 3. 1947, I 293 
ist und niemals materielle Gesctzeskralt crlang hat. Die Handlungen der Angeklagten sind deshalb objektiv rechtswidrig.a's

Das LG Frankfurt fand mit dieser Argumentation Unterstützung bei Richard Lange, der die »Überordnung der Rechtsidee über die Staatsidee forderte $^{14}$, und Gustav Radbruch, der übergeserzliches Recht über das gesetzliche Unrecht gestellt wissen wollce."s

Unabhängig davon, welcher Argumentationsvariante die Gerichte zuneigten, das Ergebnis blieb das gleiche:

-Das Ermächtigungsschreiben Hitkers vorn r.9. 1939 war also keine Rechtsnorm und hatte nicht die Wirkung einer solchen. $E_{s}$ war nichis anderes als ein Freibriel des tatsächlichen Gewalthabers an cinen bestimmten Personenkreis, das Verbrechen des Mordes zu begehen; ein Freibrief, der diese Personen braft der tatsächlichen Machr des Aussrellers de facto, aber nicht de jure vor Strafverfolgung schützte. ${ }^{16}$

Dic Abwehr des Hitler-Schreibens vom 1.9. 1939 als Rechtsgrundlage der "Euthanasieaktion w wurde so zun Paradigma der juristischen Einschätzung dieses Schreibens für die gesamie Rechespraxis seit 1945.

\section{Keine Rechefertigungs- und Schuldausscbließungsgründe}

Gesetzliche und übergeserzliche Rechtfertigungs- und Schuldausschließungsgründe wurden narirlich schon in den ersten Jahren nach dem Krieg diskuciert - und sämtlich zurückgewiesen. ${ }^{17}$

\section{a. Befehl}

Auch wenn das Hiter-Schreiben als Diensebefehl und nicht als Gesetz verstanden wurde, dem manche nordienstverpflichtete Angeklagte nach ihren Angaben nachzukommen hatten, blieb die objektive Rechtswidrigkeic bestehen.

Das KG Berlin hatte einen Betehl als Rechtfertigungsgrund mit einer zweigleisigen Argumentarion abgewehrt: Zum eineo habe - so der formalrechtliche Gesichrspunk - in der Anstalc Meseritz, in der im Rahmen der zweiten Phase der "Euthanasieaktion a Tötungen vorgenommen wurden, kein Befehl vorgelcgen, zum anderen könne sich ein Angeklagter - so der naturrecheliche Gesichtspunkt - nicht auf ein dem allgemein anerkannten Sittengesetz widersprechenden Befehl berufen. ${ }^{18}$

Den folgenden Urteilen genügte das formalrechcliche Argument; im Urteil des LG Frankfurt yom 21.12.1946 hieß es:

- Es ist anerkannten Rechts, daß derjenige, der den rechtswidrigen Befehl ausfülirr, hier also cinen Befehl zum Mord, sich nicht mit dem Hinweis, er habe nur auf Befehl gehandelt, von seiner Verantworzung befreien kann. Nach $\oint 7$ des Deutschen Beamtengesetzes ist jeder

13 LG Frankfure 11. 12. 1946, I is6.

is Lange, R. 1947, a. a. O. (Fn. 7), S. 200, r. Sp.

1) Radbruch, G.: Geseizliches Unrecht und überhescizliches Rechı, in: SJZ i (1946), S. 10 fff.

16 OLG Frankfun 12.8. 1947, 1178

17 Es kann hiee selbstversiändlich nur die allgemene Argumentauon der Genchuc bezüglich der „Euchanasiew-Taten wiedargegeben werden - der Einzellail kann von dieser Einschäzung abweichen; dem Angeklagten wird in einem rechisstazelichen Verfahren seine und niche eine allgemene Schuld zugerechnec. Obwohl z.B. die Notstandssituacion im allgemeinen für die "Euthanasıeaktiona vermeint rerden muß, könnten Einzelnc doch durch Drohungen im Sinne der Notstandsdefinution zur Teilnahme gezwunger worden sein.

is KG Berlin $24.8 .1946,1,2$. 
Beamı für die Gesetzmäßigkeit seiner Amıshandlungen verantwortlich, und kein Beamter

darf Anordnungen befolgen, deren Ausführung eine strafbare Handlung ist. $\alpha^{19}$

Das OLG Frankfurt sekundierte mit dem MilStGB:

"Blinder Gehorsam gegenüber Befehlen der Vorgesetzten ist in keiner Amtspflichr begründeL Soweir ging nicht einmal die milizärische Disziplin.*

Nacli $\$ 47$ des MilstGB vom 28.6.1872 war ein Untergebener mit der Strafe des Teilnehmers bedrohr, der einem Befehl nachkam, der sein bürgerliches oder militärisches Vergehen bezwcckte ${ }^{20}$

\section{b. Zusicherung von Straflosigkett}

Vielen Angeklagten war zugesichert worden, daß sie für ilure Taten niche zur Verantwortung gezogen werden würden, und es varen ja zatsächlich mit der Juristenkonferenz vom April $1941^{2}$ Schritte uncemommen worden, die eine Strafverfolgung der "Euthanasie - Törungen verhinderten.

"Auch [auf die Zusichenung von Straflosigkeit, S.B.] können dic Angeklagten sich nach Auffassung des Gerichts nicht mit Erfolg berufen, weil die damalige Stastsfübrung ihnen niche von Rechrs wegen, sondern lediglich aus ihrer tatsächlichen Machtstellung heraus für die Teilnahme an sırafbaren Handlungen Straflosigkeit zusichern konnte. Dic Strafbarkeit ihrer Handlungsweise war somit nicht aufgehoben, sondern lediglich in der damaligen Zeit der gerichtlichen Verfolgung entzogen. Strafwürdig war das Verhalten der Angeklagien damals wie heute, und da die Strafverfolgung nach Beseitigung bestimmzer tassächlicher Machtverhältnisse heute möglich ist, können die Angeklagten sie wegen der Strafwürdigkeit ihrer Handlungen niche mit Erfolg abwehren. ${ }^{12}$

Die Taten der Angeklageen waren also im 3. Reich de facto straflos geblieben, aber de jure strafbar.

\section{c. Notstand}

Die Angcklagten führeen vielfach aus, daß sie im Fall einer Weigerung in cin Konzentrationslager eingewiesen worden wären oder mit dem Tod bedroht wurden und damit $\$ 52$ oder $\$ S_{4}$ StGB (a. F.) erfüllt seien.

Auch dieses Rechtertigungsvorbringen wurde ihnen widerlegt. Im Einzelfall wurde gezeigt, daß die Anwerbung als »Eurhanasietäcer « freiwillig erfolgt war. ${ }^{23}$ Für die Organisatoren der „Euthanasieaktion « war die Freiwilligkeit sogar cin wichtiges Kricerium der Teilnahme gewesen:

* Denr für eine solehe Tárigkeit wollıe man auch damals gerade freiwillige Teilnehmer haben und keine dazu gepreßren Leute. Man hat sie offenbar vorher schon sehr sorgfälig ausgesucht, bevor man überhaupt an sie mit einem solchen Ansinnen herantrat. Man wollıe von vornherein keine Saboteure in dieser Aktion, $k^{2}$

Es habe zwar Leib und Leben gefährdende Drohungen gegeben,

* aber es ist zweifelsfrei, daß diese ausschließlich zur Einhalung der allen aufcrlegten Schweıgepflicht ausgesprochen worden sind. $\kappa^{1 s}$

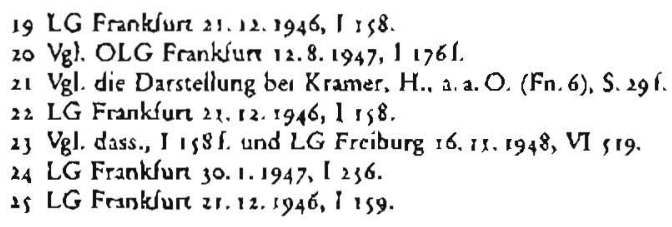


Die Weigcrung, sich an der "Akcion * zu beteiligen, habe zwar Nachteile mit sich bringen können, aber nicht die in den $\$ \$ s_{2}$ und 54 StGB angedrohten; im Einzelfall wird dies nachgewiesen. ${ }^{26}$

Das Vorliegen eines Pucativnorstands wird ebenfalls verneint, da lediglich der Bruch der Schweigepflicht bedrohe war und die Angeklageen nich schlüssig nachweisen konnten, warum sie diese Drohung mit einer bezüglich der Weigerung zum Mitmachen verwechseln konnten.

- Wenn [der Angeklagte, S. B.] Sprauer in der Haupruerhandlung glauben machen wollic, er habe seinerzeit diese verschiedenen, Drohungene durcheimandergeworfen ...., so kann das nur als eine rcichlich starke Zumutung dem Gericht gegenüber bezcichnet werden. ${ }^{27}$

\section{d. Ersetzbarkeit des Taters}

Die nationalsozialiscischen Mordeaten unterschieden sich von pgewöhnlichen * u.a. dadurch, daß die Täter bei einer individuellen Weigerung relativ sicher damit rechnen konnten, durch einen Nachfolger erserzt zu werden. Diese Ersetzbarkeit hinderte die Gerichte jedoch nicht daran, den Angeklagten die jeweils eigene Schuld zuzumessen. Das LG Freiburg führe beispielsweise zum Tatbeirag des Angeklagten Spraver aus:

"An der Kausaliëı und dem persönlichen Schuldanteil änder sich selbstverständlich dadurch nichıs, daß Dr. Linden mil Leichtigkeit einen anderen gefunden häıte, der die Sprauer zugefallenen Verlegungsgeschäftc ebenso 'gewissenhafu oder vielleicht sogar noch aktivistischer besorgi hätte; in diesem Falle hätte sich eben an Stelle Spraucrs diescr anderc heute zu verantworten, ${ }^{28}$

\section{e. Feblendes Unrechusbewußtsein/Verbotsirtum}

Viele Angeklagte argumentierten in den Gerichisverfahren als Überzeugungstäter: sie seien Anhänger einer Eurtzanasie im Sinne einverständlicher Sterbehilfe und hätren das Vernichtungsprogramm so verstanden", sie seien von dem Törungsprogramm selbsc überzeugt ${ }^{3 \circ}$ oder sie hätcen wegen eines Geseczes an seine RechtmàBigkcit geglaubi"s.

Nach der ständigen Rechusprechung des Reichsgerichts war das Bewußssein der Rechtswidrigkeit nur dann entscheidend, wenn die Rechtswidrigkeit zum gesetzlichen Tatbestand gehörte - also nicht bei Mord oder Totschlag. Dic Gerichte sahen es aber mit Ausnahme der Berliner"s' als notwendig an, das Vorliegen eines Schuldausschließungsgrundes durch fehlendes Unrechrsbewußsein zu prüfen, obwohl sie sich sonst vielfach auf die Rechtsprechung des Reichsgerichts bezogen. Sie reagierten damic auf Überlegungen der juristischen Liceracur, die schon in der Weimarer Republik das BewuBtsein der Rechtswidrigkeit für den Vorsatz gefordert hacte.

26 Z.B. LG Freiburg 16. 11. 1948, VI $\{20$.

27 Dass, VT 319. Dic Mär vom Befthlsnotseand ist anc bis heutc wirksame Schutzbehaupcung, dic «mpinsch keinen cinzigen Fall zu ihrer Beseäugung anführen kann - für sämeliche NS-Verbrechen nichi, obwohl sich ganze Heerscharen von Veneidigern um diesen Nachweis bemühten. vgl. Rückerl, A.: NS-Verbrechen vor Genche - Versuch einer Vergangenhestsbewillugung, Heidetberg 1982, S. 282.

28 LG Freiburg 16.11.1948, VI 313. Der genannie Dr. Linder war Minisecrialrae im Rechsinnenminuste rum. Spraucr hatec als Ministenalrat im Badischen Innenminiserium für die Verlegung badischer Keranker in Töeungsansealien gesorgi ("Verlegungsgeschälie a).

29 Vol. LG Frankfur 21. 12. 1946, 1140.

$30 \mathrm{Vgl}$. LG Freiburg 16. 11.1948 , VI $\{2\}$.

31 Vgl. OLG Frankfurt 12.8.1947, I 175 f

32 LG Bcrlin 25.3. 1946, KG Bcrlin 24.8.1946, a.2. O. (En. 8). 
Nach der Schließung des Reichsgerichts durch die Alliierten harte sich die Auffas-

sung der Literatur w überraschend schnell ${ }^{3} 3$ in der Rechtsprechung durchgesetzt.

Die konsequente Zuordnung des Unrechtsbewußtseins zum Vorsatz ließ jedoch geradc die Ưberzeugungsiäter straffrei, sofern die Abwesenheit des Unrechtsbewußsseins unvermeidlich war. "So bleibe der moralisch riefstehende Täter wegen einer Tat, die er eben auf Grund seiner sitclichen Fehlorientierung niche für ,Unrecht، im weicesten Sinne hält, straflos.... ${ }^{34}$ Es sei aber bei »den Verbrechen und bei den meiscen Vergehen das Vorhandensein dieses BewuBrseins in der Rege! anzunehmen; ein besonderer Beweis dieses Vorsaczbestandteils wird daher bei diesen Delikten meist nicht erforderlich sein (...).ess

Die Ablehnung eines Schuldausschließungsgrundes wegen fehlenden Unrechrsbewußtseins bei den *Euchanasien-Tärern durch die Gerichte der ersten Phase erfolgte durch ein formelles und ein materielles Argument.

Den Angeklagten wurde im Einzelfall nachgewiesen, daß sie - trotz ihres Vorbringens des fehlenden Bewußtseins der Rechtswidrigkeit - tatsächlich ein Unrechtsbewußtsein gehabt hatten:

„Der Graueshaftigkeit dieser Vorgänge [in der Zowischen- und Tötungsanstalt Eichberg, S. B.] entsprach auch voll und ganz die innere Verfassung der Angeklagten während dieser Zeit... Hiemach war ihnen die ganze Aktion sschrecklich und crschüttcrte reilweise ihr Seelenleben deran, daß zumeilen Kinder, dic getötct worden waren, den Angeklagten Schmidt und Schürg im Traume erschiencn. ... Alles dics konnte nach Überzeugung des Geriches nur dahin gewerce werden, daß sie schweres Unreche tacen. ${ }^{16}$

Das Bewußtsein der Rechrswidrigkeit müsse sich, so das LG Frankfurt, nicht auf einen bestimmten StGB-Paragraphen beziehen; es genüge «das Gefühl, Unrecht zu [un. ${ }^{37}$

Könne dem Angeklagten das Unrechtsbewußtsein im Falle von Tötung jedoch nicht nachgewiesen werden, zeuge dies von einer rechtlich unbeachtlichen Rechtsblindheit. Das Vorbringen von Angeklagten, sie hätten an eine Euthanasie im eigentlichen Sinne geglaubt, wird verworfen:

${ } \mathrm{Da}$ B der Angeklagte Dr. Schmidt an das Bestehen einer die Töung gestattenden Rechesnorm geglaubr hat, könnte ihn - vielleicht - dann entschuldigen, wenn es sich um eine Euthanasie im eigentlichen Sinn gehandelt häıt...

Nichı um eine Hilfe für die unheilbar Kranken, sondern um eine Törung im Dienste eines zynischen Utilizarismus handelte es sich (...). Dieses aber verstieß in cinem solchen Maße wider die Natur des Menschen, wider die Natur des Rechts, entbehrte also so völlig der Rechisnatur, daß ein wirklicher oder angeblicher Irrum hierüber nichr beachtet werden kann. Darf und muß schon bei jedem Menschen ein gewisser Grundstock von rechulichen Vorstellungen vorausgesetzr werden (Kohlrausch-Lange SIGB 38. Aufl. Anm. II $8 \mathrm{zu} \$ 59$ ), so kann Rechrsblindheit eines Arztes, dessen Berul das Heilen, nicht das Tören ist, gegenüber der Heiligkeit und Unverletzlichkeit jedes menschlichen Lebens, auch und gerade des schwächsten, ämsten und wehrlosen, kein Entschuldigungsgrund sein. $\alpha^{35}$

Da auch Rechrfertigungsgründe verneint wurden, wurden die Angeklagren damit in der Regel wegen Mordes verurteilt.

33 So die kritische Einschäızung von G. Radbruch in: Anmerkung zu OLG Frankfun, in: SJZ 2 (1946), S.634 H., Zirar S.634.

34 Winnefeld. H.: Bewußisen der Rechiswidagken und Vorsatz, in: DRZ 1947, S. 365 ff., Zitae S. 365, r. Sp.

35 Schónke, A.: Unrechusbewußserm, Vorsalz und Irroum, ID: DRZ 4/1946, S. 101 fl., Zital S. 102.

36 LG Frankfurt 21, 12. 1946, I 197.

37 Ebd.

38 OLG Frankfurt 1 2.8. 1947, I 176. 
Nur wenn ein Angeklagter als Täter wegen Mordes verurteilt wird, hat das Gericht bei der Festseczung des Strafmaßes keinen Spielraum: Es ist gesetzlich zur Verhängung einer lebenslänglichen Freiheirsstrafe verpflichtec. Neben der Feststellung, ob Mord oder Torschlag gegeben sei und ob es Rechterrigungs- oder Schuldausschließungsgründe gebe, spielt es ałso eine erhebliche Rolle, ob auf Beihilfe oder Tärerschaft erkanne wird.

Die Rechtsentwicklung vor 1933 gab dabei zwei mögliche Theorien zur Abgrenzung von Täterschaft und Beihilfe vor: die sogenannte eingeschränkte und die exurem subjektive Teilnahmelehre.

Im Gegensatz zur gesetzlichen Teilnahmekonzeption, die (Mit-)Täter, Anstifter und Beihelfer nach der "realen Tatnähe «, also nach objekciven Kriterien, unterscheider ${ }^{39}$, scellte sich das Reichsgericht $(R G)$ im sog. "Badewannenfall $\alpha$ auf den Standpunkt der extrem subjekciven Theorie: Eine Frau, dic im Interesse ihrer Schwester deren uneheliches $K$ ind in der Badewanne ertränkt hatte, wurde von ihm als Gehilfin, nicht als Täterin angesehen. ${ }^{\circ}$ Objektiv hatte sie zwar cigenhändig den Straftatbestand verwirklicht, subjektiv - so das RG - wollte sie die Tötung selbst nicht, sondern lediglich ihrer Schwester helfen. Das RG vertrat dabei die Auffassung, daß die Teilnahmeform - hier: Tärerschaft oder Beihilfe - sich »vornehmlich, wenn auch nicht ausschließlich, nach dem Grade des Interesses am Eriolg ${ }^{4 t}$ richte.

Aligemeiner Widerspruch gegenüber dieser Encscheidung führte zur Entwicklung einer eingeschränkten subjektiven Theorie: Wer den Tatbestand allein voll verwirklichre, sollre in jedem Fall Täter sein. Das Vorliegen von Beihilfe oder Mittäterschaft sollse dagegen weiterhin nach der subjektiven Willensrichtung des Teilnehmers entschieden werden. ${ }^{22}$

In der ersten Phase der "Euthanasie -Verfahren war die anzuwendende Theorie unstritren. Das LG Frankfurt bevorzugte von Beginn an die extreme subjektive Theorie, das OLG revidierte LG-Encscheidungen jedoch mehrfach vom Standpunkt der eingeschränkten subjektiven Theorie aus. ${ }^{4}$

Das OLG bezog sich bei seiner Argumentation auf Kohlrauschs Kommentar zum Strafgesetzbuch von 1944 (Vorbem. $24 \$ 47 \$ .167$ Anm. A) und referierte:

„Fruchcbar sei der Gedanke vom Täterwillen dann, aber auch nur dann, wenn bei Beteiligung mehrerer es sich darum handele, Täterschafi von bloßer Bcihilfe abzugrenzen. Hier solle einer, der die Tat nicht sausgeführu hat, den Tarbestand nicht verwirklicht hat, aber immerhin eine Bedingung zum Erfolge gesetzı habe, (z. 8. Wache gestanden habe) dann als Tater bestraft werden können, wenn er mit Täterwillen mitgewirkt, die Tat als eigene gewollt, nicht nur eine fremde Tat hat unterstützen wollen. Das objektive Minus werde hier durch ein subjektives Plus ausgeglichen. Aber der Gedanke sei nicht auf die Fälle zu überragen, in denen jemand den stralrechelichen Tatbestand ganz in seiner Person vcrwirkliche. Hier fehle ein soiches ausgleichungsbedürftiges Minus und daher auch der Anlaß, ein zusätzliches Element zu fordern. Wer einen anderen mit eigener Hand tötet, den tödlichen Schuß abgibt, ihm Gift in die Speisen mengt, ist Mörder, auch wenn cr es im Intercsse cines anderen, , für ihn tue.... Mit Rechr bemerke Kohlrausch, daß dieses Ergebnis mit dem Laienverstande übereinstimme. *

39 Vgl. die Ausführungen bei Roxin, C.: Strateaten im Rahmen organisatonscher Machuaparate, in: Goltdammer's Archuv 1963, S. 193 ff., Zitat S. 194 f.

40 RG 74,84 .

41 Ebd.

\$2 Vgl. Baumann, J.: Beihilfe bes eigener voller Tacbestandserlüllung (Besprechung des Staschinskij)Ureils des BGH), un: N]W 1963, S. 561 ff., Zitue S. 563 , 1. Sp.

43 Revidiert wurden: LG Frankfurt 21. 3.7947, 1 307 if. und LG Frankfur 28, 1. 1948, II $187 \mathrm{H}$.

44 OLG Frankfur 20.10.1948, [ 372 . 
Das Gericht wandte sich gegen eine Überspiczung der subjekriven Tcilnahmelehre:

$\rightarrow$ Wenn eine Rechustheoric (Willenstheorie) in ihren letzten Auswirkungen zu einem der natürlichen Betrachtungsweise widersprechenden Ergebnis fülırt, besteht kein Bedenken, sie riche bis in ihre letzten Konsequenzen zu verfolger." "s

Durch so begründere Entscheidungen der Revisionsinstanz wurden die Angeklagten in der ersten Phase überwiegend nach der eingeschränkten subjekriven Theorie verurteilt, obwohl diese schon umstritten war.

\section{Zweite Phase: Seit 1947/48 weitgehende Exkulpation der "Euthanasie - Tater}

In vier Bereichen urceilten die Laien- und Berufsrichter-16 der zweiten Phase anders als die der ersten Phase:

Die recheliche Würdigung des Tarbescandes änderte sich: In einigen Fällen wurde auf Totschlag statt Mord erkannt, die Einschäczung der Teilnahmeform verschob sich von Täterschafn auf die nun fast ausschließlich angenommene Beihilfe. Außerdem wurden nun in fast jedem Fall Rechtfertigungs- oder Schuldausschließungsgründe anerkannt: durch die Annahme eines unvermeidlichen Verbotsirnums bzw. Tarbestandsirrums und durch die Anerkennung von übergesetzlichen Schuld-/ Strafausschließungsgründen.

Die Anerkennung von Rechtferrigungs- und Schuldausschließungsgründen sowie die Erkennung auf Beihilfe waren für die Exkulpation der wEuthanasien-Tàcer am Bedeutendsten; bei den Kinderrötungen spielce die Bewertung der Törung als Totschlag statt Mord eine große Rolle.

Die Urteile der zweiten Phase enthiclten auch Argumentationsstränge, die in der Tradition der ersten Phase standen: Nach $1947 / 48$ erklärten alle Gerichte die „Euthanasieakrion \& weiterhin für rechrswidrig, weil die Hitler-Ermächtigung keine Legicimationsgrundlage für die Anstaltscötungen sei. Dazu wurden die formalrechtlichen und/oder naturrechtlichen Begründungen vorgetragen, die in den Jahren zuvor entwickelt worden waren. Auch das Vorliegen der geseczlichen Rechrferrigungs- und Schuldausschließungsgründe wurde abgelehnt.

Diese Anknüpfung an die Argumentacionsstrukturen der ersten Phase veránderte jedoch - da die subjektive Tatseite mitslerweile grundsäzzlich neu bewertet wurde das Ergebnis von milder Verurteilung oder Freispruch nicht. Die zweite Phase der juristischen Bewertung der "Euchanasier-Taten dauer bis 1974, d.h. bis zum letzten rechtskräftigen "Euthanasie "-Urteil des BGH'77 an. Fast die gesarnte bundesrepublikanische Rechesprechung ist also dieser zweiten Phase zuzurechnen. ${ }^{18}$

\footnotetext{
4) Ebd.

46 "Euthanasic $\alpha$-Prozesse fanden vor Schourgencheen stact, die meheheldich mit Laveoricheem besetat waren.

$47 \mathrm{BGH} 20.3 \cdot 1974,2$ StR $589 / 72$.

${ }_{4} \$$ Das kürzlich ergangene Frankfurter „Euchanasıex-Uneil (vgl. dic regelmäßige Berıchuerstatrung der Franklurer Rundschau sm Sommer vergangenen Jahres) konnte nicht mehr analystert werden. Es stcllt in gewisser Weise den Beginn cuner dritien Phase der *Euthanasiea-Rechtsprecinung dar, da dic Angeklagten un Gegensatz zur zwerten Phase wegen Bcihilfe zum Mord venureilt wurden.
} 


\section{a. Die Rechtsprechung 1948: Übergesetzlicher Notstand als persönlicher Strafausschließungs- oder Rechtfertugungsgrund}

$194^{8}$ standen zum ersten Mal Personen vor Gericht, die in sog. „Zwischenanstalren ${ }^{49}$ bzw. der staatlichen Gesundheitsverwaltung beschäfrigt gewesen waren ${ }^{10}$. Das LG Koblenz sprach am 4. 10. 1948 einen Anstaltsarzt und einen Anstaltsdirektor frei, da sie sim Zustande der P lichterkollision das Menschenmöglichste getan hätten, um größeres Unhcil abzuwenden. «" Die Pflichtenkollision wurde so beschrieben:

"Darf das Bemühen, das Leben einzelner, ja vieler Menschen zu retten [indem sie erwa Patienten vom Transpor in die Tötungsanstalten zurückstellicn, S.B.], dazu führen, selbst dabei mitzuwirken, anderc dem Tod zu überliefern? Hier seızı zum mindesten ein tragischer Pflichtenkonflikt ein, hicr muß der Gehilfe - und nur von eıncm solchen, nicht vom Täter selbst soll hier gesprochen werden - Schuld auf sich nehmen, um großeres Unheil zu verhüren. $\star^{s^{2}}$

Das Koblenzer Urreil bezog sich ausdrücklich aul zwei schon sehr früh (1 947 und Fcbr. 1948) erschienene Arrikel des Godesberger Strafrechesprofessors Hellmuth von Weber, der den Gedanken der Pflichtenkollision entwickelt und festgestelle hatce:

n[M]an (wird) anerkennen müssen, daß oft der größere moralische Mut zum Ausharren in der Stellung und zur Mitwirkung bei hemmender Befehlsausführung gehört und daß durch dicse von verantwortungsbewußren Männern bewiesenc Halrung unter nationalsozialistischer Herrschaft oft viel Unheil verhindert worden ist.""s

Er hatte darür plädiert, die Lösungen, die Angeklagee in der nrragischen Pflichrenkollision $\propto$ nach gewissenhafter Prüfung gewählt hatten, der strafrechtlichen Beurteilung gänzlich zu entziehen:

"Die Lösung derarriger Konflikte kann nur vom Absoluten her im Gewissen gefunden werden; sie muß der Einzelne mit seinem Gott susmachen. Die Rechtsordnung gibt für ihre Losung keinen Maßstab. Wegen dicses Mangels ihrer Zuständigkeit solltc aber auch der Entschluß, zu dem sich jemand nach emster Gewissensprüfung in einem solchen Konflikt durchgerungen hat, keiner strafrechtlichen Beurteilung unterworfen wcrden. «s

Das LG Koblenz nahm Webers Gedanken der ernsthaften Gervissensprüfung auf und fertigte eine Lisce mit sechs Voraussetzungen an, die gegeben sein mußten, um Beihilfe zu Törungshandlungen zu entschuldigen. Zu den Voraussetzungen zählten u. a. das Vorhandensein ciner echeen Zwangslage, aus der es keinen anderen Ausweg gab, um das Leben wenigstens eines Teils der Gefährdeten zu rerten, die Beschränkung der Beihilfe auf das »alleräußersce Mindescmaßs, die unter Gefährdung der eigenen Sicherheit fortaufend durchgeführte Sabotage, die

49 Sest Herbse 1940 wurden die Opfer zur Tarmung zunachse in "Zivischenansialien a gebracht, um von dorr nach einiger Zert in dic Totungsanstalicn transportien zu werden. Das Personal kannie in der Regel Ziel und Zweck der Transporre, für die es durch Ausfullen von Meldebögen und Vorbcreitung der Kranken wesentliche Hilie lessecte.

so In der Zeıt zuvor waren vor allem Personen angcklagt gewesen, die un Tötungsanstaleen selbst gearbeıte hatten.

sI LG Koblenz 4.10. 1948, VII sif., Zital VII 16.

s2 Ebd., VII is.

s) Weber, H. v.: Die strafrecheliche Verantwortlichkeit für Handeln auf Befehl, in: MDR :/1948, S. 34 fl., Zitat S. 4 I , I.Sp.

if Weber, H.v.: Die Pflichtenkolhsion im Strafrecht, in: Festschrife fir Wilhcim Kissclbach, Hamburg ${ }_{1947}$, S. 233 If., Zital S. 250. 
win solchem Umiange ausgeübt worden sein (muß), daß die Zahl der durch sie gerelteten Mcnschenleben wenigstens in etwa in einem angemessenen Verhältnis zur Zahl der geopferten Menschenleben stehi. «"s

Das LG Koblenz leitete aus der Situation der Pflichtenkollision einen persönlichen Srrafausschließungsgrund für die Angeklagten ab, der zu ihrem Freispruch führe, obwohl der geseczliche Tarbestand der Beihilfe zum Mord in etwa rooo Fällen verwirklicht worden war.

Der persönliche Strafausschließungsgnund, der die Tat weiterhin für objektiv rechtswidrig erklärte, den Angeklagten jedoch wegen seiner persönlichen Situation straffrei ließ, ging dem LG Münster niche weit genug: Es wollce die Angcklagten in scinem Verfahren gerechifertig $\imath$ wissen. ${ }^{36}$ Die Pflichtenkollision sei derart gewesen, daß die Angeklagten, zwei Angestellte der Gesundheitsverwaltung und ein Arzt, auf die cine oder andere Weise Schuld hätten auf sich laden müssen. Am 5. 3.1949 hob der Oberste Gerichtshof der Britischen Zone (OGHBZ) jedoch das Urteil des Schwurgerichss Münster vom 20. 10. 1948 auf.

Obwohl der OGH die Annahme eines persönlichen Strafausschließungsgnundes befürwortete, nahm er die Revision zum Anlaß, um seine grundlegende Kricik an der Anwendung der Lehre vom übergesetzlichen Norstand auf die „Euthanasieakion « darzulegen.

Zunächst stellte er fest, daß es kemeswegs ausgemacht sei, ob der bestimmende Beweggrund für die beiden Angeklagten P. und S. wirklich der gewesen war, möglichst viele Patienten zu retten. Ihre Partei- und SA-Amter sowie die freiwillige Teilnahme an der „Euthanasicaktion« stünden zunächst Rechtfertigungs- und Schuldausschließungsgründen entgegen. 57 Diese seien erst nach genauer Prüfung anzunehmen.

Der OGH mochte es nicht ins Belieben des jeweiligen Täters stellen zu ensscheiden, ob er sich in der Situation des übergeserzlichen Notstandes (bzw. des persönlichen Strafausschließungsgrundes) befinde, denn damic sei das Verbor der Teilnahme an ciner Straftac durchlöchert. Selbst ein Notstandstärer müsse abwägen, ob gegebenenfalls der Eingriff in fremde Rechte das geeignete Mitrel zur Rettung aus der eigenen Gefahr sei; um so mehr gelce das für denjenigen, der andere aus der Gefahr retten wolle. Der Notstandstäter habe seine Handlungen nicht am eigenen Maßsuab auszurichten, sondern am gesetzlichen. Anderentalls sei er unter Umständen zur Teilnahme an einer fremden Straftat berechrigt, was rechtlich unannehmbar sei. ${ }^{38}$ Im Rahmen der »Euthanasieaktion« war der "allgemein geltende Maßstab», der geseczliche, eindeutig; Der Grundsatz der Pflichtenkollision war auf die zahlenmäßige Abwägung von (zu retrenden und preiszugebenden) Menschenleben nicht anwendbar, weil Menschenleben gegeneinander nicht abwägbar sind.

Das RG, auf dessen Rechtsprechung sich das LG Münster bezogen hatte, war bei der Annahme der Pflichtenkollision immer davon ausgegangen, daß zwei unterschiedlich zu bewertende Rechtspflichten kollidierten "9: In dicsem Falle war der Täter gerechtertigt, wenn er dic böhere Rechtspflicht erfüllte, indem er die mindere verletzı. Der Täter verletzıce dann nämlich lediglich das geringerwertige Rechtsgut

3s LG Koblenz 4. 10. 1948, VII 16

36 Das Ureil des LG Munster liegr mur niche vor; es wnurde aus der Ureilszusammeníassung um Uncil des OGHBZ v. 5. 3. 1949 rekonstruiert, 5. dazu unten. Ein ähnliches Ureil wie das des LG Mùnster erging aाT 24.11, 1948 vom LG Düsseldorf, III 465 if.

57 OGHBZ 5. 3. 1949, XI 708 If., Zicar XI 7Is.

s8 Ebd., Xl $>16$.

59 Z. B. RG 61, 242; abgewogen wurde das Leben der Leibesfrucht gegen das der Schwangeren: Der Arzt sei bei einer Abereibung gerechefertige, wenn das Leben der Schwangeren aul dem Spiel stehe. 
und schürzte das höherwertige. Das Werceverhältnis der Rechesgüter ergab sich aus dem Strafrahmen der sie schützenden Rechtssätze.

Der OGH hielt die Pflichtenkollision i.S. des RG bei den "Euthanasien-Tätern nicht für gegeben, weil sie nicht ein höherwertiges Rechtsgut auf Kosten des geringerwertigen geschützt hatcen. Ihre Rechtsgüter waren gleichwertig gewesen:

"Die Angeklagten haben sich gegen das Gcsetz entschieden. Dieses wernet zwar das menschliche Leben höher als die Leibesirucht oder die körperlichc Unversehrehcit. Dagegen wertet es das menschliche Leben gleichmäßig, und das mit vollem Recht. Jeder anderc Maßstab wäre unerưäglich und entwürdigend. ...Daß das bloße günstige Zahlenverhälınis zwischen den Gererteren und den zur Retung Aufgeopferen deren Törung für sich allein niemals zu rcchrfertigen vermag, ergibr sich danach von selbsi und außerdem auch bereits aus dem Umstande, daßis nichr der - vom Täter gar nicht beherrschuc - später eintretende, mehr oder weniger zulällige Erfolg über die Rechtmäßigkeit der Tat entscheiden kanm. ${ }^{\text {6o }}$

Zudem habe es überhaupt keinen wirklichen Widerstreit von Rechtspflichten keinen tragischen Pflichtenkonflikr - gegeben, denn:

↔[hre Arztoflicht, ihren Kranken mit ärzclichen Mitteln nach Krafzen zu helfen und ihre allgemeine Staatsbürgerpflichı, sich von Strafraten fernzuhalten, geboren ihnen rechlich nicht, sich an der Aktion zu beteiligen. Den Angeklagten wäre im Gegentcil rechtlich kein Vorowurf erwachsen, wenn diese Pfliclıcen sie von jeder Beteiligung abgehaluen häuen. ${ }^{61}$

Es gab also rechtlich nur eine Pflicht: sich von Mordtaten fernzuhalcen.

Nachdem der OGH mit klaren Woren das Vorliegen eines übergeserzlichen Notstandes bestritten harce, weil dieser u. a. Menschenleben gegeneinander abwäge, entwickelte er einen persönlichen Strafausschließungsgrund, der durch die Hintertür diese kritisierce Folge bei der Annahme des übergesetzlichen Notstandes wieder hereinließ.

* Stand ein.... Arzt vor der Wahl, jede Teilnahme an dem Verbrechen abzulehnen, beiseirezuzreten, dadurch williährigeren Krälten Raum zu geben und so alle seinc Patienten, soweit sie zum Tode bestimmt waren, widerstandslos zu opfern, oder aber möglichst viele von ihnen durch ıätiges, nur ihm mögliches Eingreifen zu reuten, freilich ausschließlich um den Preis seiner entferaten Mitwirkung bei der Vernichtung der Unrettbaren, so darf die Entscheidung für das Letzterc, wenn sie nach sorgfältiger Prüfung und Abwägung allein aus sirtlichen Beweggrunden geschah, gerechterweise strafrechdich nicht unbeachtet bleiben. ${ }^{6 r}$

Der Oberste Gerichtshof sah eine solche Handlung jedoch im Gegensatz zur Lehre vom übergesetzlichen Notstand nicht für rechtmäßig an und ließ den Angeklagten deshalb nur unter bestimmten Voraussezzungen, die erwa denen des Koblenzer Urteils vom $4 \cdot 10.1948$ entsprechen, persönlich straffrei. ${ }^{63}$

Die Voraussetzung, daß die Widerstandshaltung des Angeklagten

*sich also nachgewiescnermaßen... mic Erfolg ausgervirkt haben (muß) " und die "bloße, wenngleich bewiesene Absicht dazu nicht (genügt), $\alpha^{6_{4}}$

führe praktisch doch wieder zur Abwägung von Menschenleben. Denn der " $\mathrm{E}_{\mathrm{r}}$ -

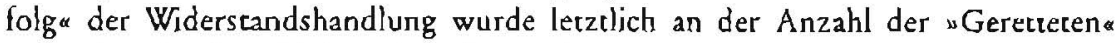
gegenüber der Anzahl der "Geopferren« bemessen.

Dagegen umging die Konstruktion des persönlichen Strafausschließungsgrundes die übrigen vom OGH am übergeserzlichen Norstand kritisierten Folgen der Nichtbeachtung der Rechtspflicht, sich von Straftaten fernzuhalten und der tendenziellen nachträglichen Berechigung zur Teilnahme an einer fremden Strafcar, indem er die

6o OGHBZ $\{\cdot 3 \cdot 1949, \mathrm{Xl}>\times 6$

6r Ebd.

62 Ebd., XI 718.

63 Ebd., XI 719

64 Ebd. 
Handlungen der angeklagten Ärzte einer Zwischenanstalt weirerhin als rechtswidrig und scluuldhaft einstufte.

Insgesamc läßt sich das Urteil des OGH vom $5 \cdot 3 \cdot 1949^{65}$ also als ambivalent charakterisiercn: Einerseits wehrte es die rechtlich unzulässige Konstruktion des übergesezzlichen Notstandes ab, andererseits entging es jedoch dem Problem der unterschiedlichen Bewertung von Menschenleben durch die Anerkennung eines persönlichen Surafausschließungsgrundes nicht.

\section{b. Die Rechisprechung 1949-52: Ubergesetzlicher Schuldausschließungsgrund}

Die Rechtsprechung des Obersten Gerichcshofes der Britischen Zone wurde in der juristischen Literatur breit diskutiert und nahezu einhellig abgelehnt. Die Strafrechtswissenschaftler plädierten fast sämtlich für die Anerkennung eines übergeseczlichen Schuldausschließungsgrundes. ${ }^{66}$ Auch die Gerichce lehnten die OGH-Rechrsprechung eindeutig ab.

Das LG Münster (Urteil vom 29.8.1949) und das LG Düsseldorf (Urreil vom 27. 1. 1950) waren an die OGH-Rechtsprechung gebunden und sprachen die Angeklagten wegen Vorliegens eines persönlichen Strafausschließungsgrundes frei. Das LG Düsseldorf versäumte dabei nicht hervorzuheben, daß es das Handeln des Angeklagten $C$. für gerechrfertigt halte (dieser hatte für die Verlegung rheinischer Kranker in Zwischenanstalten gesorgt):

* Wenn er dann aber diese anzuerkennende siltliche Konflikrlage in beachelichen und berviesenen sittlichen Erwägungen nur mit dem Ausharxen aul seinem Posten pflichigemäß lösen zu können glauble und dieser siulichen Entscheidung seines Gewissens gefolgı ist, so entfallt damit allein schon das Rechı jeglichen Vorwurfes nact menschlichem Maße. a

Indem seit 949 die rechtliche Schuld der Angeklagten verneint und ihre mögliche sittliche Schuld in außerrechrliche Bereiche des je eigenen Gewissens abgedrängt wurde, war tendenziell der cinzelne Handelnde zum Richter über sich selbst geworden: Nur er konnte entscheiden, welche Handlung spflichtgemäßu war objektive Kriterien darüber gab es nicht mehr.

Gleichzeirig wurde prakisch der unverbrüchliche rechrtiche Schutz des Lebens nachträglich aufgehoben - die einzig wirklich (rechts-)pflichtgemäße Alternative, der Rückırit von der Teilnahme an der *Aktion", wurde in den Urreilen überhaupe niche mehr diskutiert.

Die Grenzen, die der Oberste Gerichtshof hatte setzen wollen, wurden durchbrochen: "Geretrete« und "Geoplerte" wurden ausgezählt, um die Schuld der Angeklagten auszuschließen. Um möglichst viele „Geretrete nachzuweisen, wurden zunächst eirmal alle Geisteskranken als schon verloren angesehen, um Zurückstellungen von Kranken in den Zwischenanstalten als »Retcung $\propto$ definieren zu können. Ofr wurde dann so lange mir den Zahlen* (d. h. Menschen) jongliert, bis ein für

\footnotetext{
6) Ein ähnliches Ureeil erging vom OGHBZ am 23.7.1949, III 530 ff.

66 Vgl. zu OGHBZ 5. 3. 1949 dirckt: Schmidh, E.: Anmerkung zu OGHBZ vom 5. 3. 1949, in: SJZ 8/1949, S. ss9ff., Welzel. H.: Anmcrkung zu OGH v. 5- 3. 1949, in: MDR 1949, S. 370 if., Peters, X.: Zur Lehre von den persónlichen Strafausschließungsgründen, in: JR 1949, S. 496 ff. Peters war der einzige, der Jem OGH in Jer Konscrukuon des persönlichen StralausschlicBungsgrundes zusumme. Zv OGHBZ 23.7.1949: Hartung, F.: Anm. zu OGHBZ vom 23.7.1949, in: NJW 1950. S. Iss. Zur OGHRechesprechung allgemeın: Reinicke, D.: Zur Rechusprechung des Obersten Gericheshofes für dic bnusche Zone uber die Anwendbarkeil des Grundsatzes in dubio pro reo ber Strafausschließungsgruinden, in: MDR i9so, S. 77 fi., Klefisch, T.: Die nationalsozialisusche Euthanasie im Blickleld der Rechesprechung und Rechislehre, in: MDR 1990, S. 298 (f., Welzcl, H.: Zum Norscindsproblem, in: Zertschrife für die gesamec Staaesrechts wussenschatr 63 (1950), S. 47 H.

67 LG Dusseldorf 27. 2 . 1950, VI 67.
} 
den Angeklagten günstiges Verhältnis von "Geretreten « und "Geopferten « heraus$\mathrm{kam}^{6 \mathrm{~g}}$

Das "Mitmachen" von Ärzten und Anstaltspersonal bei der Organisacion von Transporten in dic Tötungsanstalten wurde in der Argumentacion der Gerichce tendenziell zur sirtichen Pflich, indem davon ausgegangen wurde, daß der Angeklagte die Retcung einiger nicht hä̈te bewerkstelligen können, ohne dic übrigen preiszugeben. ${ }^{69}$

Die Beweggründe des Anstalsspersonals beim Zurückstellen von Patienten waren selten objekciv nachweisbar. ${ }^{\circ}$ Sie wurden jedoch von den Gcrichten in den allermeisten Fällen als Widerstandshandlung ausgelegt. Blickt man auf die Gesamtheic der Gerichesurteile, so kommt man zu dem Ergebnis, daß anscheinend jeder der angeklagten Ärzte und Pfleger aus Zwischenanstalten Widerstand geleiscet hat.

\section{c. Die Rechisprechung des BGH 1952 und 1953: Vom übergesetzlichen Schuidausschließungsgrund zum entschuldigenden Irrium über das Vorliegen eines übergesetzlichen Schuldausschließungsgrundes}

Der Bundesgerichrshof hatre sich in zwei Urteilen 1952 und 1953 mir der Lehre vom übergeserzlichen Schuldausschließungsgrund zu befassen." Er grenzce dabei die Lehre vom übergesetzlichen Schuldausschließungsgrund ein und überführte sic prakcisch in die Lehrc vom Verborsirrtum.

In seinem Urteil vom 28. ir. 1952 sah sich der BGH an die Rechtsprechung des $\mathrm{OGH}$ gebunden und führee dessen Argumentation forr.

Der rechtfertigende, übergeserzliche Norstand wurde deshalb mit den Überlegungen des OGHBZ abgeletint.

"Das Vorliegen cines rechıfertigenden, übergesetzlichen Notstandes hat der erste Revisionsrichter [OGHBZ, S. B.] mit bindender Wirkung für das gegenwärige Verfahren verneint, weil die Angeklagten sich nicht in einem Widerstreit von Rechespflichten befunden haben, und weil auch kein rechtlich beachtlicher Konflikt zwischen gesetzlich verschieden bewerteten Rechesgürern bestanden hat. ...

Selbst wenn man aber wenigstens eine sıttliche Pflicht der Angeklagten bepahen würde, die ihnen anvertrauten Kranken auch außcrhalb des ärzllichen Aulgabenkrcises vor dem ihnen durch starliche Maßnahmen drohenden Tode zu schüızen (...), so müßıe eine solche Verphichrung gegenüber sämtlichen Anstaltspfleglingen angenommen werden, von denen die Angeklagten nach den Urteilsfescscellungen jedoch nur den kleineren Teil retceten, während sic zur Tötung der größercn Anzahl beitrugen.

Ausschlaggcbend war daher in jedem Fall die Rechrspflicht zur Befolgung des Tötungsverbots. " ${ }^{72}$

Der BGH präjudizierte damit jedoch nicht eine Verurteilung der Angeklagten, sondern entwickelte eine Lehre, die man die wLehre vom Verborsirrtum wegen Rectungsabsicht" nennen könnte, und die wie die abgelehnce Lehre vom übergeserzlichen Schuldausschließungsgrund zum Freispruch führen konnte. Der Verborsirrtum wegen Rettungsabsicht wurde so beschrieben:

w Wenn die Angeklagten sich aber verpflichtet fühlten, möglichst viele dem Tode verfallene Kranke zu retten, läß sich nicht ohne weiteres ausschließen, daß sic es nicht als Unrecht

68 Z. B. LG Hannover 29. 7.1950, VII $87 \mathrm{ff}$, LG Koblenz 28.7. 1950, VII sff.

69 LG Koblenz 29.7. 1948, XXII s8, fh. LG Koblenz 28.7. 1950, 3. 1. O. (Fn.68), LG Koln 24. 10. 1951, nach: BGH ig. s. 1953, XII s $8 \mathrm{ff}$.

70 Ein Ansulesdirektor konnce ctwa daran interessicr sein, dic besser zahlenden Prvatpatsenteri in der Ansualt zu behaleen und die ubrigen mabzugeber.

7) BGH 28. 11. 1952, XY 723 ff. und BGH 19.5. 1953. XII 58 ff.

72 BGH 28.11.1952, XI 725f. 
ansahen, wenn sie zu diesem Zweck zu der nach ihrer Überzeugung unvermeidlichen Vernichtung der übrigen Gcisteskranken in enternter Weisc beitrugen. $x^{7}$

Die juristische Einschärzung cines solchen Irrams über das Vorliegen eines übergeserzlichen Schuldausschließungsgrundes ist im Ergebnis dieselbe wie die bei der Annahme eines übergesetzlichen Schuldausschließungsgrundes: Sie entschuldigr den Angeklagten:

"... [D]em [darf] kein Schuldvorwurf gemacht werden, der in einem wirklichen oder vermeinilichen Widerstreit zwischen wirklichen oder vermeintlichen Forderungen des Gesetzes und wirklichen oder vermeintlichen Fordenungen seines Gewissens der Suimme des Gewissens folgt, vorausgesetzt, daß er nach dem Maß seiner geistigen und situlichen Kräfte sein Gcwissen angespannt hat. सt $^{7}$

So blieb der BGH theorerisch in der Tradition des kritischen Teils der $\mathrm{OGH}$ Rechtsprechung, der die Rechrspflicht zur Befolgung des Törungsverbors gestützt hatce.

Er entwickelte aber gleichzeitig mit der Kombination von Irrtumslehre und Lehre vom übergesetzlichen Schuldausschließungsgrund cine Argumentation, die die prakrischen Handlungen der Täter völlig ins Belieben ihrer subjektiven sitclichen und gewissensmäßigen Einschätzung der Siruation stellte: Hatte der Täter sein Gewissen nur gehörig angespannt, so konnce er am Massenmord teilnehmen, um "Schlimmeres zu verhüten", oder auch davon zurücktreren - cntschuldigt war er in jedem Fall.

Die letzten Säıze des zweiten BGH-Urteils waren geradezu eine Einladung an die Landgerichte, nun doch statt nach übergesetzlichen Schuldausschließungsgründen nach fehlendem Unrechtsbewußtsein zu suchen:

¥... auch der vorliegende (Fall bieter) keinen hinreichenden Anlaß, die Frage zu erörtern, ob cs Lebenslagen gibt, in denen jemand, der sich gegen das Strafgesetz vergangen har, infolge cines nicht im Strafgesetz vorgeschenen Schuldausschließungsgrundes straffrei bleiben muß. Es ist kaum cin Fall denkbar, in dem dann der Täter das Bewußtscin der Rechtswidrigkeit... gehabt haben könnte. «7s

Und die Einladung wurde angenommen: Die drei auf die BGH-Entscheidungen folgenden Urcile, die ihren Freispruch noch auf den übergesetzlichen Schuldausschließungsgrund scüczten, caten dies nur in Kombination mit dem Verbotsirrtum: Die Angeklagien häten ihr Verhalten nicht als Unrecht betrachtet, da sie um die Rettung möglichst vieler Kranker bemüht gervesen waren. ${ }^{76}$

\section{Entwicklung neuer Rechtfertigungs- und Schuldausscbließungsgründe: Die Irrtumslebre}

Schon in der ersten Phase hatren sich die Gerichte mit dem Argument auseinandersetzen müssen, die Angeklagten hätten nicht gewußt, daß ihre Handlungen Unrecht seien. Sie hätten kein Unrechtsbewrßsein gehabt und über die Rechtnäßigkeit ihrer Taten geirrt. Die Gerichte wehrten diese Verteidigung $a b$, indem sie den Angeklagten ein Unrechtsbewußtsein nachwiesen oder es ihnen zurechneten. Die Tötung von Menschen wurde in den ersten Nachkriegsjahren als ein so schweres Verbrechen angesehen, daß die Rechtsblindheit eines Angeklagten, der sie aus ideologischen oder anderen Gründen als erlaubt ansah, für rechtlich unbeachtlich erklär wurde.

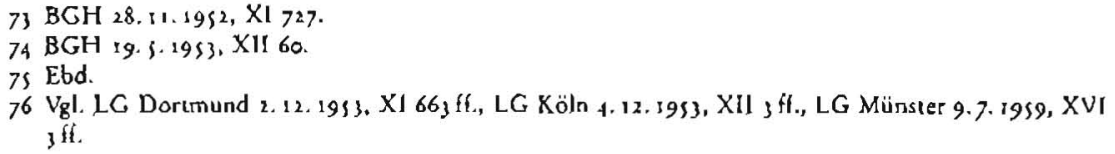


Die Zurechnung von Unrechtsbervußrsein war eng mit naturrechtlichen Überlegungen verknüpft:

„Ganz allgemein stellt sich aber die Frage, ob niche die Bcjahung eines durch keinen Gesetzgeber antasibaren Kernbereich des Rechts - eines Minimums an Menschenrechten wie des Rechrs auf Leben, auf physische Freiheit und auf Gleichheit aller ohne Ansehen der Person - notwendigerweise auch die Bejahung cines Kernbereichs von Rechts- und Unrechesbewußssein bei einem jeden nach sich zichen muß. ${ }^{\text {th }}$

1946 hatte es noch geheißen:

"Bei den Verbrechen und bei den meisten Vergehen ist das Vorhandensein dieses Bewrußseins [Unrechr zu tun, S. B.] in der Regel anzunehmen .... $\alpha^{\text {in }}$

Diese Voraussetzung war nach 1947/8 nicht mehr gegeben, der Irrtum über die Rechtmäßigkeir von Massenmord wurde in der Rechesprechung wie ein Verbotsirrrum im Orónungsstrafrecht behandelt:

-Fehir dem Täter infolge cines entschuldbaren Tar- oder Rechesirnums das UnrechrsbewuBtsein, so bleibr er straffrci. ...

Als entschuldbar wird cin Irrtum immer dann anzusehen sein, wenn der Täter alles getan hat, was billigerweisc von ilhm verlange werden kann. $u^{\text {BO }}$

Was von den Angeklagten nach 1947/48 "billigerweise verlangt « wurde, war niclut viel. Folgr man der Rechtsprechung, hatte es im Dritten Reich viele Gründe gegeben anzunehmen, daß vielfacher Mord an Kranken und Behinderten erlaubi sei.

\section{a. Verbotsirnum oder: Das Lebensrecbt von Bebinderten ist kein allgemeines Siliengeselz ${ }^{81}$}

In der zweiten Phase bezog man sich weiterhin auf die Naturrechrsargumente, um die Rechrswidrigkeir der Hitler-Ermächtigung vom Okrober 1939 zu begründen, lehnte aber gleichzeitig und im Widerspruch dazu mir historischen Hinweisen die Anerkennung eines Narurreches auf Leben ab. Ein Urtcil dcs LG Götringen hat hierfür exemplarischen Charakter:

"Ob die Vernichtung lebensunweren Lebens ${ }^{\text {a }}$ absolut und a priori als unsitrlich und gegen eine höherc Rechcsordnung verstoßend anzusehen ist, kann schon zweifelhaft sein. Insbesondere bestehen gegen den Ausgangspunkı des... Ureils des Oberlandesgerichts Frankfur am Main ${ }^{83}$ (...) erhebliche Bedenken, daß es sich hicrbei um einen Verstoß gegen naturrechelich evidente Sütze handele. ... Für besonders schwere Fälle geistigen Todest oder körperlicher Mißbildungen (Monstra) sind solche Tötungen schon in vorchristlicher Zeit crörtert worden Es ist bekannt, daB im klassischen Altenum die Vernichtung lebensunwerten Lebens durch Aussetzung und dergleichen eine gewisse Rolle gespielt hat (...). Seneca spricht... wie selbstverständlich von der Ausmerzung ${ }^{8 s}$ Minderweriger (...). Auch Plato gibt einem ähnlichen Gedanken Ausdruck...

Man wird kaum behaupten kónnen, daß die Ethik Platos und Senecas sittlich tieler stehr als diejenige des Christentums. Unter dessen Einfuß ist dann allerdings die Vernichrung lebensunwerten Lebens überwiegend abgelehnt worden...

Indessen hat Thomas Morus in seincr Utopia $(1,16)$ die Einschläferung ${ }^{82}$ unheilbar Krankes (wenn auch niche gerade gcistig Minderwertiger) in einem Sinne diskutiert, daß sich der soziale

77 Bauer meint hier die Gleichlicit aller vor dem Gesetz; die Gleschbest aller war nach nie ein erstrebenswerics Ziel.

78 Bauer, F.: Das gescizliche Unrecht des NS und die deuesche Stratrechispilege, un: Gedachtnisschrifr für G. Radbrich (Hg. A, Kaufmann). Goiringen 196R, \$. zo, ff., Zital S. 30 .

79 Schönkc, 1. a. O. (Fn. 3s), S. 102, I. Sp

80 Ebd., S. 102, r. Sp. Schörlee sah den Umachivung kommen und kommentiere ilan abwaigend.

8I Die verschicdenen Vananten des Verborsimums tauchen in den Uneilen meist in einer Mischung. selten alleın aul. Zweı Varianten werden bier ausführlicher dargestelit.

82 Hicr üird NS-Terminologic übemommon!

8) OLG Feankiurt 12.8.194\%, a.1. O. (Fn. 8) 
Gcdanke ankündigt (...). Auch Dr. Martin Luther bat sich in seinen Tischgesprächen über die Tötung eines, Wechselbalges، zu Dessau in zustimmendem Sinne geäußert. $a^{84}$

Hier wurden gelegenrliche $\ddot{A} u ß e r u n g e n$ aus früheren Jahrhunderren verglichen mit einem in der Geschichte einzigartigen Massen-Mord an über 100000 Behinderren.

Die Richter griffen auf Autoren zurück, deren Argumente von denen der Nationalsozialisten und ihrer Wegbereiter nicht weit entfernt waren: Luther begründete seine Zustimmung zum whomicidium « damit, daß das « Wechselbalg $\propto$ ohne Seele $\mathrm{sei}^{i s}$ - was anderes sind die ngeistig Toten * bei Binding und Hoche, den intellekruellen Vordenkern der NS-»Euthanasie $\alpha$ ?

Morus stimmte der „Einschläferung unheilbar Kranker " aus dem »sozialen Gedanken heraus zu. "Sozialer Gedanke «, das klingt altruistisch und ist in Wahrheir ein Euphemismus: Nach Morus solleen sich Kranke u.a. dann selbst töten, wenn sie anderen zur Last fielen. ${ }^{\mathrm{S6}}$ Was anderes ist das nationalsozialistische Nützlichkeitsdenken, das den "Wert der Behinderten nach den Kosten, die sie der »Volksgemeinschaft « verursachten, bemaß? Um keine Irritation aufkommen zu lassen, wurde eine wichrige Bestimmung des Thomas Morus unterschlagen:

"Gegen seinen Willen aber bringen die Utopier niemanden ums Leben .... ${ }^{8}$ ?

Schließlich wurde auf die Schrift Bindings und Hoches von 1920 eingegangen $^{89}$ :

"... [A]usschlaggcbend ist allein, daß auch anderswo [im Ausland, S. B.] und unter rechtsstaatlichen Verhälmissen und Garanvien die Beseitigung äußerst uefstehender idiotischer Menschen crnsthaft erörnert worden ist. $x^{89}$

Zum einen ignorierte das Geriche, daß die „Euthanasieakrion « weit über das hinausging, was selbst Binding und Hoclie angestrebt hatten, zum anderen wurde die zeirweise "Erörterung" der Menschentönng, die überdies mehrheitlich abgelehnt worden war, als Legitimationsgrundlage für ihre Durchführung angesehen.

Die angeklagten Leiter der Kinderfachabteilung Uchtspringe, die aufgrund von Berliner Tötungsermächeigungen 130 bzw. 60 "geistig minderwertige Kinder vernichtet $" 9{ }^{\circ}$ hatren, wurden von der Anklage des Totschlags freigesprochen : Sie hätcen nicht wissen können, daß die Auswahl der Kinder nicht nach dem geregelten Verfahren Binding/Hoches vonstatten ging. Im übrigen sei die "Erlösung aus Mitleid a mit den Kindern geschehen.97 Die Angeklagten hätten sich deshalb in einem Verborsirrtum bezüglich der Rechtmäßigkeit von „Euthanasiemaßnahmen* befunden.

\section{b. Verbotsirrtum oder: zu ungebildet und autoritätsgläubig, um das Untecht zu erkennen}

Speziell bei Angeklagten, die in abhängiger beruflicher Position getötet hatten, d. h. besonders bei Pflegern und Schwestern, wurde ein weiteres Argument für das Vorliegen des Verbotsirrtums entwickelr: Sie seien als Nichcakademiker zu ungebidet oder - aufgr und ihrer dienstlichen Stellung - zu autoritätsgläubig gewesen und hätcen darum den Anordnungen der Vorgesetzten blind vertraut.

84 LG Götungen 2.12.1953, XI 735fl., Zitar XI 7581.

8 , Ebd., XI 759.

86 Morus, T.: Uropia (1516), Leipzig 1982, 5.93.

87 Ebd., 5.94.

88 Auf die Schrilt des ehem. Reschsgerchespräsidenten K. Binding und des Psychtatrieprofessors Hoche *Die Freigabe der Vernichtung lebensunwerren Lebens. Ihr Ma $B$ und ihre Form $\propto$ (Leipzig 1910), hanem sıch nauonalsozialistische "Euthanasie $\alpha-I$ deologen und -Tàter berufen.

89 LG Göıtungen 2.12.1953, Xi 762.

90 Ebd., XI 764.

9r Ebd. 
Dieses blinde Vertrauen wurde ihnen jedoch nicht zum Vorwurf gemacht - es wurde geradezu als narürlich und unvermeidlich angesehen, daß Untergebene autoritärshörig und ungebilder sind:

* Allcrdings ist niemand der eigenen Gewissensprüfung und der eigenen Verantwortung vor dem Gesciz enthoben. Daher würde ein kritikJoses Vertrauen in das Handeln der vorgeserzıen Ärzı die Angeklagte dann nicht vor Sirafc schüızen, wenn sie das Unrecht ihres Tuns erkannt hätc oder doch hätte erkennen können. Die Angckłagte war zur damaligen Zeit bereits is Jahre alt. Sie hatte also eine gewisse Lebensreife und Erfahrung. Sie war jedoch nach Herkunft und Schulbildung ein einfacher Mensch. Als Anstaltsptlegerin hat sie eine solide Ausbildung genossen. Dabei hat sie insbesondere gelernt, sich dienstich unuerzuordnen und in den Ärzten und sonstigen Diensrvorgeseızren Persönlichkeiren zu schen, dic unbedingte Autorität genießen und deren dienstiiche Anordnungen zu befolgen sind. Diese Umstände waren aber besonders geeignet, in der Angeklagicn das Bewußtsein zu begrüinden, daß shre von den Ärzten vorgeschriebene und geforderte Mithilfe bei den Einschläferungen in Einklang mit Gesetz und Rechc stünden. «"2

Zum Argument vom Verbotsirrtum läßt sich allgemein sagen, daß es sämtliche Sicherungen durchbricht, die die ersie Phase gegen leichtertigc Freisprüche aufgebaut hatte. Zrwas wurden diese Sicherungen für die objektrve Bewertung der -Euchanasieaktion« weiterhin anerkannt (ein Gesetz zur "Euchanasieaktion « hat es nicht gegeben, die Hitler-Ermächtigung war rechtswidrig usw.), aber in der subjektroen Bewertung zunichte gemacht: Die Anerkennung der objektiven Geltung des Rechtsschurzes und des $\$ 211$ StGB hatce keinerlei prakcische Auswirkungen mehr: Die Angeklagten hatten fakrisch jeweils selbsi entschicden, ob sie sich nach dem Tötungsverbot zu richten hatten oder es durchbrechen duriten. Dieses Vorgehen der Gerichte bedeutete praktisch die Aufhebung des Lebens-Rechtsschutzes für Behinderte in der NS-Zeit. ${ }^{93}$

\section{Milde Strafen durch die Beibilfekonstruktion der extrem subjekiven Theorie}

Führten der übergesetzliche Schuldausschließungsgrund und der Vcrborsirrtum zur Entschuldigung der Táter und zum Freispruch, so ermöglichten die Beihilfekonstruktion der extrem subjekriven Theorie und die Erkennung auf Totschlag statt Mord vergleichsweise geringe Strafen für "Euthanasica-Täter.

In der ersten Phase wurde noch nach der eingeschränkten subjektiven Theorie geurteilc, in der zweiten Phase fası durchgängig nach der extrem subjektiven Theorie, die - weil sie die kaum objekcivierbare Willensrichtung des die Tat eigenhändig Ausiührenden zum Haupckritierum macht - für außerstrafrechrliche Einflüsse besonders anfällig ist. ${ }^{94}$ In der ersien Phase war daher der Mord aus Gleichgültigkeit kein Hindernis, Tärerschaft anzunehmen, wenn er eigenhändig ausgeführt worden war. In der zweicen Phase wurde bei Mord aus Gleichgültigkeit regelmäßig nur Beihilfe angenommen. Dies wiegt um so schwerer, als es bei Massenverbrechen nur wcnige Personen gibt, die sie anordnen, aber viele, die sie

92 LG Düsseldor 7.2.1953, X 339 ff., Zirat X 345.

9) Es gab außer den zweı hier dargestcllen Vetbotsirnumsargumenuationen zablreiche weitere: so der Verbousirtum wegen ciner allgemeinen Ausnahmesituxion im Krieg (so z. B. OGHBZ 23.7. 1949, III 130(f), wegen der Annahme einer Euchanasse im eigentlichen Sinne (z. B. LG Dissseldorf 3r. 5. 1951, VIII 433 if.) ader der Irrum ïber das Vorliegen enes ubergeseczlichen SchuldausschlieRungsgrundes, wie ihn der BGH anerkannte (vgl. die Darstellung der BGH-Rechisprechung oben).

94 Vgl dazu auch Kruse, F.: Zweierle Maß für NS-Täer? in: Redakion Krulisclie Jusuy (Hg.): Der Uncechisstane - Reclut und Juseiz im Nationalsozidismus, Frankfurt 1979, S. igolf. 
ausführen, wie etwa Schwestern und Pfleger. Waren diese zwar eigenhändig, aber

lediglich auf Anordnung von Vorgesetzten tätig gewesen und daher nur wegen Beihilfe verurteilt worden, so mußte logischerweise der Täterwillen bei den Leitern der Mordorganisation zu finden sein und bei diesen zu Verurceilungen wegen Täterschaft führen.95 Die 1968 und 1970 vor Gericht gestellten vier leitenden Funktionäre der »Euthanasie -Organisation wurden jedoch wegen Beibilfe zum Mord zu Freiheitsstrafen zwischen 7 und ro Jahren verurteilt.

Auch sie hätten - so das LG Frankfurt ${ }^{96}$ - keinen eigenen Täterwillen gchabt, sondern nur den Willen der politischen Staatsführung ausgeführt - obwohl sie, wie das Gericht selbst feststellt, eigene [deen zur besseren Tarnung der Krankenrötungen entwickelt und durchgesetzt hatten"7;

"In subjektiver Hinsicht läßt sich nicht feststellen, daß die Angeklagten ,Vorberg und Allers, S. B.) das der Mordaktion zugrundeliegende Motiv, nämlich einen Nutzen zu erzielen, zum Motiv ihres eigenen Handelns gemacht haben odcr daB sie selbst den Opfern gegenüber feindselig gesonnen gewesen sind. Es kann nocht ausgeschlossen werden, daß die Angeklagten sich zur Mitwirkung an der Mordaktion lediglich durch die wenn auch zu mißbilligende Vorstellung veranlaßt sahen, sie schuldeten den damaligen Machthabern Gchorsam, zumal keine Anzeıchen dafür ersichtlich sind, daß die Angeklagıen sich zur Mitwrkung gedrängt oder irgendein persönliches Interesse an der Durchführung der Tötungsaksion gehabt haben. $x^{\text {ss }}$

Wer blieb nun als Täter übrig? Die amaßgebenden Initiatoren wie Hitler, Bouhler, Brandt, Brack, Blankenburg, Heyde und Nitsche $\$, 99$ deren Täterschaft juristisch nicht mehr fesrstellbar war, da die Genannten gestorben oder von Alliierten verurteilt waren.

\section{Milde Strafen durch die Annabme von Totschlag statt Mord bei den Kindertötungen}

Die an den Kindertötungen Beteiligten wurden nur halb so oft wegen Mordes verurteilt wie die Gesamtzahl der Täcer. ${ }^{160}$

Die Tarbestandsmerkmale Heimrücke ${ }^{101}$ und Grausamkeir ${ }^{102}$ galten bei den E $\mathrm{I}_{-}$ wachsenen- wie bei den Kindertötungen tendenziell als nicht gegeben. Niedrige

9s Àrze wurden meist wegen übergeseizlicher Schuldausschließungsgründe freegesprochen.

96 LG Frankfun 20,12.1968. Ks 2/66 (GSLA). Das Uneil wurde vom BGH im 27. 10. 1972 bestangr (2 S(R ros/70).

97 LG Frankfurt 20.12. 1968, a. a. O. (Fn. 96), S. iss.

98 Ebd., S. 116

99 Ebd.

ro Eigene Berechnung. Die Berechnungsgrundlage bilden simtliche verfugbaren s Euthanasıea-Uncile von 1946-1974. Als statustischer Bezugspunkt dienten 105 der 108 in den Jahren 1946-1974 wegen

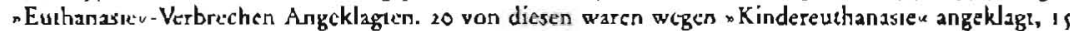
murden verurteilt, bei 6 von ihnen wurde Mord angenommen (= c3. 40\%). In der Gesamtgruppe der wegen „Euthanaste $\alpha$-Verbrechen Venureilen $(=43$ Personen) wurdc dagegen bes 31 Mord angenommen ( $=c a \cdot 76 \%$ ).

101 Heımeucke wurde of abgelehne, weil geısıg Kmnke nıchr täuschbsr seren (z. B. LG Göıngen 1.12. 1953, 3. a. O. (Fn. 84), weil dic Tötungtn heimlich, aber miche :ückssch ausgehuhrt worden sesen (z. B. LG Munchen I 15.3. 195), VIII 283 fi.) oder wcil dic Eltern angeblich zugesumme hatten oder

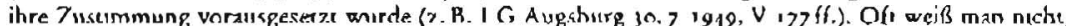
welche Kranken die Gerichre vor Augen hateen: Die meısten waren durchaus tz̈uschbar. Fusi alle Elecm waren überdies mit der Toung iheer Xinder nicht esnverstanden.

102 Das Mlcrkmal der Grausamkell wurde außerst selten angenommen. Dagegen wurden die Gastötungen vom LG Frankfure 1968 (Uncil vorn 20.12.1968, [Fn. 96] S. rog) als xene der humansten Toungsarten « bezerchnet. Auch die Tötungen durch Gifunjektionen oder Tablettengaben wurden allgemein niche als grausam angesehen, obwohl sic mcist zu einer Schwachung des Körpers und Lungconcntzundung vor dem Tod fuhren. 

Erwachsenentötungen:

- Die Beweggründe der Angeklagten [Schwestern, S. B.], die anfänglich Icdiglich dern Aufurag des Dr. P. nachkamen und die im weiteren Verlauf der Akvion, naclidem ihnen der Zweck ibrer Täwgkeit offenkundig geworden war, aus, was ihnen geglaubc werden muß, rein menschlichen Gründen den Tod der Kinder als eine Erlösung für diese betrachceten, können nicht als niedrig angesehen werden. ${ }^{103}$

Die Gerichte der zweiten Phase faßten den Anstaltsmassenmord an Kindern of als Mitleidsgeste auf. ${ }^{\text {ra }}$ Daneben wurde der Gedanke der Tötung behinderter und deshalb minderwertiger Menschen als achtenswerces Motiv anerkannt:

„Der Angeklagre handelte als Anhänger des Euthanasiegedankens, der von jeher und zum Teil auch heute noch, durchaus ernstzunclimende Verfechter hat, was nicht verkannt werden darf, also aus weltanschaulichen und menschlichen Grïnden, Mordlusi oder sonstige niedrige Beweggründe veranlaßeen ihn nicht, Tötungen vorzunchmen. $x^{105}$

Die "Euchanasieaktion unterscheidet sich an diesem Punkt deutlich von anderen NS-Massenverbrechen: Wurden hier aus "welcanschaulichen Gründen« Behinderte getötet, so war dies oft genug kein Grund für die Annahme niedriger Beweggründe, während Törungen aus Rassenhaß ais niedrig angesehen wurden. ${ }^{106}$ Die Vorgcschichte der "Euthanasieaktion " und die Ideologie des Sozialdarwinismus wurden zustimmend dargestellt:

"Wahrend des I. Weltkrieges sah er [der Angeklagte, S. B.] in seiner Eigenschaf als Anstaltsarze an der Nervenbeilanstalt in Homburg/Pfalz, wie die Kranken an Hungerödemen unter den fürchterlichsten Verhältnissen wie die Fliegen wegstarben. Meistens waren es die arbeiten. den Kranken, während die unheilbaren und nichtarbeirenden Kranken, die imenschlichen Leichen, am Leben blieben. ${ }^{10}$

Die (verkappten und offenen) Anhänger von Krankentötungen verurteilten diejenigen, die sie ausgeführt hatcen, nur zu geringen Strafen.

\section{Hypothesen für die Erklärung der Exkulpation von "Euthanasiex- Tatern}

Die Gerichte hatten seit 1947/48 durch die Entwicklung neuer SchuldausschlieBungsgründe und die Anwendung der extrem subjektiven Teilnahmelehre einen

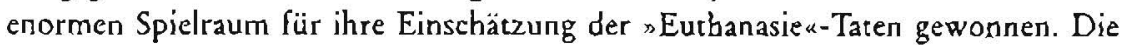
zunehmende Subjektivierung der Frage der strafrechtlichen Ahndung der NS"Euchanasiex fungierte als Eingangstor für außerjuristische politische Einschätzungen. Das Argumentieren mit dem übergesetzlichen Schuldausschließungsgrund verhalf nicht allein einzelnen Angeklagten zu einem Freispruch. Es diente nicht nur dazu, die spezifische Situation des (vermeintlichen oder wirklichen) wtragischen Pflichtenkonflikts « angemessen juristisch zu erfassen. Dies hatte nämlich bereits der vorn Obersten Gerichtshof der Britischen Zone entwickelte persönliche Strafausschließungsgrund erreicht. ${ }^{108}$

103 LG Munchen I 21.7. 1948, III 29, Zitat III 29.

104 Dies finhre of́ zur Anerkennung eines Verbossirrums wegen der Annahme der Euthanasie um eigentlichen Sinnc.

105 LG München I 15. 1. 19\$1, VIII 294.

106 Bongard, A.: Die Uneerscheidung zwischen Mord und Totschlag, in: RaL, M. L. a.: Die Justiz und die Nazs - Zur Strafverfolgung von Nazismus und Neonazismus seit 1945, Frankfun 1979, S. 79 (fi., Zilat S.814.

107 LG München I 19.3.1991, VIII 284

108 Dies hat ein Befurworer des ubergesetzlichen Sehuldausschließungsgrundes inih fesigestell, vgl. 
Das Ziel des rechtscheorecischen wie rechtspraktischen Übergangs vom persönlichen Strafausschließungsgrund zum übergeserzlichen Schuldausschließungsgrund kann m. E. nur die allgemeine (und niche lediglich ausnahmsweise) Exkulpation derer scin, die vielleicht nicht zu den politischen Organisatoren der Massenmorde gehörcen $^{109}$, sich aber dem an sie herangetragenen Ansinmen der Beihilfe oder Mistäterschaft bci Mord nicht entzogen. In der Mehrzahl der Fälle (der OGHBZ härte einen persönlichen Srrafausschließungsgrund nur für wenige Ausnahmen für möglich gehaiten) dürfte nämlich das Moriv zum »Mitmachen « nicht die Verhinderung "größeren Übels^ gewesen sein, sondern die Unannehmlichkeiten bei einer Weigerung. Daß beim »Mitmachen « auch vielleicht einige Kranke gerettet werden konnten, diente überwiegend der Gewissensberuhigung.

Diese Gewissensberuhigung sollte über 1945 hinaus weitergeführt werden. Dabei wurde die Reihenfolgc von Haupt- („Mitmachen") und Nebenhandlung („Rettung ) vertauscht: Nun war dic »Retcung * der Kranken die Haupthandlung, die Mirwirkung am Anstaltsmord war pure Nebensache.

Vergegenwärtigı man sich, in welch großem Ausmaß der Medizinerstand - über Gcsundheitsverwalter, Anstaltsärzte und -psychiater sorvie Wissenschaftler - in die "Euthanasicakrion" cinbezogen war" ${ }^{\prime \prime}$, so wird dic Bedeutung dieser "Verkehrung deutlich. Die Ärzte verstanden sich im Driten Reich wic in der Bundesrepublik als eine der Spitzen der »normalen « bürgerlichen Gesellschaft, die mir Verbrechen nicht in Verbindung gebracht werden konnte. Diese Selbsteinschätzung wurde von den Gerichten übernommen und durch geringc Freibeitsstrafen bzw. Freisprüche aufrechterhalten.

Nichr der persönliche Strafausschließungsgrund, sondern der übergesetzliche Schuldausschließungsgrund mache die Beihilfe zum Mord oder sogar dic Täterschaft bei Mord praktisch ungeschehen, indem sie sic entschuldigre. Darin lag das Ziel des Übergangs zum übergeserzlichen Schuldausschließungsgrund.

Die häufige Annahme von Verbotsirrtum zeigt ebenso wie die häufige Erkennung auf Torschlag bei den Kindertörungen, daß die Gerichte der zweicen Phase eine bescimmte Affinicät zu einer »Euthanasie“ im nacionalsozialistischen Sinne hatten. Die verständnisvoll legitimierten Kindertötungen wurden dabei mit den aus der NSIdeologie bekannten Mitleids- und Zweckmäßigkeits" "Mäntelchen verschleiert und shoffähigu gemachr. Bezüglich der Behinderten befand sich die bundesdeutsche Justiz seit $1947 / 48$ in einem barbarischen Zustand, der Behinderten das Lebensrecht in bescimmien Ausnahmesituationen oder sogar genereli absprach.

Die subjektive Teilnahmelehre, die besonders bei "Tötungen auf Befehl* zum Tragen kam, bevorzugte nichı nur nachträglich das aucoricäre Bevwußtsein, sondern basierre darauf: Ihr lag eine simperative Rechisauffassung ${ }^{1 r x}$ zugrunde, die die Urform des Rechts in Befchlen, in Geboren oder Verboren, erblickre. Eine Scraftar wurde weniger als Verletzung eines bestimmen Rechtsguces ${ }^{13}$ begriffen denn als

Rentcke, D.: Zur Rechesprechung des Obcrsten Gerscheshofes für dic britusche Zone..., a.2. O (Fn. 66), S. 78, 1.Sp.

109 Mit dem übergesetzlichen Sclubldausschließungsgrund wurden vor allem Ärzıe aus Zwıschenansıalten und Fachleute aus der scatlichen Gesundheitsverwaltung freigesprochen.

n10 Vgl. die Darstellung ber Klec, E.: „Euthanasıc* Im NS-Staat ..., a. a. O. (Fn. 5), S. 39s (f. und ders.: Was sie caten - was sie wurden - Arztc, Jursien und anderc Beteiligte am Kranken- oder Judenmord, Frankfurt 1986, S. 144 ff.

111 Z. B. durch dic Anerkennung eines lrnums uber das Tötungsverbot in nknsenhalien Kriegszeiten*.

112 Der Begriff stammi von Gcrmann, vgl. Radbruch, G.: Anmerkung zu OLG Frankfur, a. a. O. (Fn. 33), S. $6 ; 4$.

II Der Begrifl des * Rechesgutes a verliert sene Konturen, wenn er im Natonalsozıalismus mit - Gemennschaftsinteressena identifizier und dadurch prakesch "cnimaterialisierta wird, vgl. Frommel, M.: Welzels finale Handlungslehre. Eine konseriacive A nowort auf das nationalsozialistusche Willenssiral- 
Ungehorsam gegenüber der rechtsetzenden Gewalt. Die Einschätzung staatlich angeordneter oder geduldeter Verbrechen bereitece dann besondere Schwierigkeiten.

Gustav Radbruch beurteilte diese Auf(assung 1947 so:

„... Unrecht ist Unrecht nichı, weil es verbolen Ist, sondern es wird verboren, weil es Unrecht ist. Der Imperativ, die Bestimmungsnorm, ist nur eine sckundärc Form des Rechts, bestimmt, den einzelnen die Erfüllung des Reches ins Gewissen zu schrciben; die Urform des Reches abcr ist die Bewertungsnorm, das Werturteil der rechtsetzenden Macht, durch das cinc Handlungsweise für ungerechı und antisozial erklär wird. a"s

Nach Radbruchs Rechtsauffassung wird - nach einem Wercurceil - lediglich negativ bestimmt, welche Handlungen rechtswidrig sind. Nicht der verbrecherische Wille führt zur Strafbarkeit, sondern bestimmte, pönalisierte Handlungen, die ein schürzenswertes Rechtsgut verlerzen.

Nach der mimperativen Rechtsauffassung \& bestimmt das Recht nicht negativ, welche Handlungen wegen ihres Unrechrsgehalts zu unterbleiben haben, sondern stellt posicive Verhaltensnormen auf, denen der Bürger zu folgen hat. Folgr er ihnen nicht, so ist er strafwürdig, weil er ungehorsam ist, nicht weil er ein bescimmtes schüczenswertes Rechtsgut verletzc. Eine soiche Rechtsauffassung ist autoritär.

Für die Teilnahmelehre hat die jeweilige Rechtsauffassung Konsequenzen: Nach Radbruchs Rechtsauffassung werden. Täterschaft und Beihilfe nach dem Grad der Rechtsgutverletzung unterschieden, also stärker nach objekriven Kriterien"'s, während sie nach der mimperaciven Rechtsauffassung "nach subjektiven Kriterien unterschieden werden, nämlich nach dem Grad des «Ungehorsams gcgenüber dem Geserz:

"Wie die Enescheidung gegen das Gesetz, der böse verbrecherısche Wille, zum Tütcr machı, so liefen konsequenı auch die Wiilensstärke das Abgrenzungsmerkmal zwischen den verschiedenen Graden der Mitwirkung an einem Verbrechen. ${ }^{116}$

Verbrechen aus "Gleichgülcigkeit " oder in Erfüllung "dienstlicher Pflichten" sind mach dieser Rechtsauffassung, die nicht primär nach der Reclicsgutverlerzung fragt, kaum exakt faßbar, weil ihnen der sverbrecherische Antrieb « fehlt.

Durch die extrem subjekuve Teilnahmelehre wurde die Mitläuferhaltung im Nationalsozialismus nachträglich akzeptiert: Diejenigen, die das Mordsystem geleitet oder in Gang gehalcen haten, erscheinen als bloße Rädchen in einem ihnen fromden Syscem.

rechr - oder die Legende von der ä̈berwindung des Werneucralismus a im Strafrecht, in: Reifner, U. Sonnen, B.-R. (HG.): Strafiustiz und Polizes im Drilten Resch, Frankfuru New York ig8 + , S. 36 II 114 Radbruch, G.: Anmerkung zu OLG Frankfurt, a. a. O. (Fn. 33), S.634.

i) Roxin schreibe: rach der weslen Tatnähe $v_{\text {, }}$ l. Roxin, C.: Serafiaten im Rahmen organisatonscher Machtapparate, 2. 2. O. (Fn-39), S. 195

116 Baumann, J.: Beilsilfe bes eigencr voller Tatbescandscrtullung, 4. a. O. (Fn. 42), S. 563. I. Sp. Ähnliche Äußerunger finden sich in "Euchanasie Ureilen, vgl. LG Frankfur 28.1.1948, 3.a.O. (Fn.43), II 196. 